\title{
CMOS CIRCUITS FOR THERMAL ASPERITY DETECTION AND RECOVERY IN DISK-DRIVE READ CHANNELS *
}

\author{
Aaron Lee \\ Dept. of ECE \\ UC Davis \\ (now with AMD)
}

\author{
Paul Hurst \\ Dept. of ECE \\ UC Davis
}

\author{
Kiyoshi Fukahori \\ TDK Semiconductor \\ Mountain View, CA
}

\begin{abstract}
A thermal asperity detection and recovery scheme for a disk-drive read channel is presented. The circuit design and simulation results for two key blocks, the AC coupler and its tuning loop, in a $0.5 \mu \mathrm{m}$ CMOS process are described.
\end{abstract}

\section{INTRODUCTION}

In a modern disk drive, the read head flies so close to the media surface that they occasionally come into contact with each other. When this happens, a large voltage is generated at the output of the read head preamp, and this is called a thermal asperity (TA) [1]. Modern read channels include a TA detection and recovery circuit as part of the front end [2], which is shown in Fig. 1.

The read signal from the preamp, $V_{i n}$, which is a bandpass signal, is input to the AC coupler (ACC), which is a high-pass filter. Then the variable gain amplifier (VGA) amplifies the signal to give the output signal $V_{\text {out }}$. This output signal is fed to the back end of the read channel to extract the data. Also, $V_{\text {out }}$ is fed into a 1-pole low-pass filter that has a low bandwidth and outputs the average value of $V_{\text {out }}$, which is referred to as the baseline. The baseline is a constant (zero) under normal operation.

However, when a TA occurs, the baseline experiences a large shift as shown in Fig. 2. A large baseline shift makes data detection difficult and can cause bit errors, as the large amplitude change typically causes clipping in the VGA. The amplitude of the baseline can be used to detect a TA event. If the amplitude of the baseline is larger than some threshold, the comparator output goes high in Fig. 1. Then a signal from the state machine reduces the AC coupler time constant which reduces the time required for the baseline to return to zero. When the baseline falls to within a threshold of zero, the time constant in the AC coupler is returned to its nominal value.

Fig. 3 shows the schematic of the AC coupler. Each NMOS transistor is biased in the triode region with an onresistance $R_{f}$. Assuming an ideal opamp, the circuit has a zero at dc, a pole at $-1 / R_{f} C_{f}$, and a high-frequency gain of $-C_{i n} / C_{f}$. In normal operation, it should have a well controlled time constant so it passes the entire read signal. We chose $R_{f} C_{f}=32 \mathrm{~ns}(-3 \mathrm{~dB}$ frequency $=5 \mathrm{MHz})$. A tuning circuit is used to set the on-resistance of the transistors by controlling $V_{\text {tune }}$ to set the time constant, as described in the next section.

The AC coupler includes two additional transistors in parallel with each M1' that are not shown in Fig. 3. These transistors are identical to M1', and they are normally off with their gates tied low. If a TA is detected, the gates of these transistors are connected to $V_{\text {tune }}$, reducing the time constant by a factor of three to more quickly return the baseline to zero.

A folded-cascode opamp [3] is used in this AC coupler to achieve high gain, large bandwidth and large output swing. With $C_{i n}=C_{f}$, the opamp must have a unity-gain bandwidth that is about equal to the data rate. Here, the target data rate was $800 \mathrm{Mbps}$, so this opamp dissipates a significant amount of power. To save power, the tuning circuit does not use a replica(s) of the AC coupler.

\section{TIME CONSTANT TUNING CIRCUIT}

A tuning circuit sets the on-resistance $R_{f}$ in the AC coupler so that $R_{f} C_{f}$ is constant despite changes in $C_{f}$ due to process variations. For a NMOS device in the triode region with $V_{D S} \ll V_{G S}-V_{t}$, the on-resistance $R_{f}$ is given by

$$
R_{f} \cong \frac{1}{k^{\prime} \frac{W}{L}\left(V_{G S}-V_{t}\right)}=\frac{1}{g_{m}},
$$

so the on-resistance can be varied by adjusting $V_{G s}$. Eqn. (1) also shows the on-resistance $R_{f}$ is about equal to $1 / g_{m}$ of an identical, saturated transistor with the same gate and source voltages [3]. Therefore, if we can generate gate and source bias voltages in a reference circuit to give $C_{f} / g_{m}=32 \mathrm{~ns}$ and apply them to M1', then $R_{f}=1 / g_{m}$ and $R_{f} C_{f}=32 \mathrm{~ns}$. The tuning scheme used here is based on this idea.

The reference tuning loop uses a Gm cell in feedback as shown in simplified form in Fig. 4 [4]. A constant current $I_{R}$ is pushed into the Gm cell, producing a voltage $V_{\mathrm{o} 1}=I_{R} / g_{m}$. $\phi_{1}$ and $\phi_{2}$ are two non-overlapping clocks with frequency $f_{\text {clock}}$. During $\phi_{1}$, the capacitor $C_{1}$ is charged to $V_{\mathrm{o} 1}$. During $\phi_{2}$, the charge on $C_{1}$ is transferred to capacitor $C_{H}$. Also, a constant current $N I_{R}$ is drawn from $C_{H}$. The average value of the opamp output, $V_{f 2}$, is used to tune the

\footnotetext{
* This research was funded by UC MICRO Grant 01-084, with support from Broadcom, Intel, Metalink, TDK Semiconductor and TI.
} 
transconductance of the Gm cell.

When the loop reaches steady state, the charge injected onto $C_{H}$ by $C_{1}$ during $\phi_{2}$ equals the charge removed from $C_{H}$ by $N I_{R}$ in one clock period. Therefore $V_{\mathrm{o} 2}$ is periodic and $V_{f 2}$ is constant. In steady state,

$$
C_{1} V_{o 1}=N I_{R} T \text {, }
$$

where $T=1 / f_{\text {clock }}$ is the clock period. Substituting $V_{\mathrm{o} 1}=$ $I_{R} / g_{m}$ into (2) and simplifying gives

$$
\frac{C_{1}}{g_{m}}=N T=\frac{N}{f_{\text {clock }}},
$$

where $g_{m}$ is the transconductance of the Gm cell. Hence, $C_{1} / g_{m}$ depends on the clock frequency $f_{\text {clock }}$, which is derived from a crystal oscillator, and $N$, the ratio of DC currents that can be accurately defined in a CMOS process.

A more detailed block diagram of the tuning loop is shown in Fig. 5. The clock frequency is $6.25 \mathrm{MHz}$ and the DC current ratio $N$ is 0.2 . Using these values in (3), $C_{l} / g_{m}$ $=32 \mathrm{~ns}$. If $C_{l}$ has the same nominal value as $C_{f}(=0.6 \mathrm{pF})$ in the AC coupler, then $g_{m}=18.8 \mu \mathrm{A} / \mathrm{V}$. With $I_{R} \leq 5 \mu \mathrm{A}$ in Fig. 5 , the nominal differential output voltage of the $\mathrm{Gm}$ cell is $\leq 0.53 \mathrm{~V}$. To have a large $g_{m}$ tuning range, the $\mathrm{Gm}$ cell should allow an output swing of $>1.0 \mathrm{~V}$.

The opamp in the switched-capacitor (SC) integrator in Fig. 5 is a folded cascode. Its DC gain is about $75 \mathrm{~dB}$ and its gain-bandwidth product is about $120 \mathrm{MHz}$; both are sufficient for this application. The SC low-pass filter (LPF) in Fig. 5 has a bandwidth of about $800 \mathrm{kHz}$. Because its bandwidth is not critical, a passive first-order filter is used to save power. A differential-to-single-ended converter converts the SC LPF output to voltage $V_{f 2}$ that sets the tail current and $g_{m}$ of the Gm cell.

\section{THE Gm CELL}

Fig. 6 shows the schematic of the Gm cell in Fig. 5. It consists of a differential $g_{m}$ stage, a common-mode feedback (CMFB) circuit, and an output buffer. The $g_{m}$ stage consists of transistors M1-M7. M1 and M2 are diodeconnected as in Fig. 5. M3-M6 form current sources controlled by the CMFB circuit. M7 is the tail current source with its current set by $V_{f 2}$. Thus, the $g_{m}$ of M1 and M2 is controlled by $V_{f 2}$.

M201-M204 form the CMFB circuit. Because M1 and M2 are diode connected, the common mode (CM) output voltage can be controlled indirectly by controlling the voltage $V_{S}$ at node $\mathrm{S}$ (the source of M1 and M2). The CMFB circuit forces the voltage $V_{S}$ to be the same as the applied CM reference voltage $V_{\text {ref }}=1.2 \mathrm{~V}$.

M101-M103 generate $V_{\text {avg }}$, the average of $V_{\text {out }}$ and $V_{\text {out. }}$ M101, M102 and M103 are copies of M1, M3 and M5 (or M2, M4 and M6), respectively. M5, M6 and M103 mirror the current in M204. These currents are pushed into diode-connected M1, M2 and M101. When the input current is zero $\left(I_{R}=0\right.$ in Fig. 5), $V_{G S 101}=V_{G S 1}=V_{G S 2}$ because $I_{D 5}=I_{D 6}=I_{D 103}$ and $\mathrm{M} 1=\mathrm{M} 2=\mathrm{M} 101$. In this case, the average gate-to-source voltage of M1 and M2 is just $V_{G S 1}$, which equals $V_{G S 101}$. When $I_{R}$ is not zero, $V_{G S 1}$ is increased by $I_{R} / g_{m}$ and $V_{G S 2}$ is decreased by the same amount. Therefore the average of $V_{G S 1}$ and $V_{G S 2}$ is still equal to $V_{G S 101}$ and is equal to the DC bias value of the gate-to-source voltage of M1. So

$$
V_{\text {avg }}=\frac{V_{\text {out }+}+V_{\text {out }-}}{2}=V_{S}+\frac{V_{G S 1}+V_{G S 2}}{2} .
$$

Since the CMFB circuit forces $V_{S}=V_{\text {ref }}$, we have

$$
V_{\text {avg }}=V_{S}+\frac{V_{G S 1}+V_{G S 2}}{2}=V_{r e f}+V_{G S 101}
$$

Therefore the gate voltage of M101 is $V_{\text {avg. }} V_{\text {avg }}$ is buffered by the voltage buffer formed by M301-M307 that outputs $V_{\text {tune }}=V_{\text {avg }}$. Circuitry that is not shown generates bias voltages $V_{\text {bias }}$ and $V_{\text {casc }}$.

\section{USING THE TUNING LOOP TO SET $R_{f} C_{f}$}

The purpose of the tuning loop is to set the time constant in the AC coupler in Fig. 3. To do this, $C_{1}$ in Fig. 5 is equal to (a copy of) $C_{f}$ in Fig. 3. Also, the on-resistance $R_{f}$ of M1' in Fig. 3 is made equal to $1 / g_{m}$ of M1 in Fig. 5. This is achieved by making M1' a copy of M1 and matching the gate and source voltages of M1 and M1'. The gate voltage of M1 is $V_{\text {tune }}$ and its source voltage is $V_{r e f}$. If the gate of M1' is connected to $V_{\text {tune }}$ and the CM output voltage of the AC coupler is set to $V_{r e f}$ as indicated in Fig. 3, then M1 and M1' are biased identically.

One limitation of this tuning scheme is the limited tuning range of the $g_{m}$ value. Another limitation is that this tuning approach depends on matching between $C_{f}$ and $C_{1}$ and between M1 and M1'. Also, tuning accuracy is limited by offsets in the SC integrator and Gm cell in Fig. 5.

\section{SIMULATION RESULTS}

The tuning loop in Fig. 5 and the AC coupler in Fig. 3 were simulated using SPICE. In the simulations, all switches and opamps were realized with MOS transistors, and $V_{D D}=$ $3.3 \mathrm{~V}$. The simulations used $0.5 \mu \mathrm{m}$ CMOS models, and the $R_{f} C_{f}$ product was found for different $C_{1}=C_{f}$. Simulation results are given in column 2 of Table 1 for $I_{R}=5 \mu \mathrm{A}$.

Column 2 shows there is a $2 \%$ change in $R_{f} C_{f}$ when $C_{1}$ changes from $1 \mathrm{pF}$ to $0.7 \mathrm{pF}$. However, $R_{f} C_{f}$ is less than the desired value of $32 \mathrm{~ns}$. Possible explanations are that (1) does not accurately describe the relationship between $R_{f}$ and $1 / g_{m}$ in this sub-micron process and/or parasitic capacitance in parallel with $C_{1}$ due to the MOS switches in Fig. 5 effectively increases $C_{1}$ so that it is not equal to $C_{f}$. For this application, the slightly low value of $R_{f} C_{f}$ is acceptable. However, when $C_{1}$ is less than $0.6 \mathrm{pF}$, the variation in $R_{f} C_{f}$ becomes unacceptably large. This error is caused by nonlinearity in the $\mathrm{Gm}$ cell. The linear range of the $\mathrm{Gm}$ cell decreases as $g_{m}$ decreases. To show that reducing nonlinearity reduces this error, the simulations 
were repeated with $I_{R}=1 \mu \mathrm{A}$. With a smaller input current to the $\mathrm{Gm}$ cell, nonlinearity is less of an issue and less change in the $R_{f} C_{f}$ product is expected for small capacitance. This is verified by the simulation results in column 3 of Table 1 , where the variation in $R_{f} C_{f}$ remains small for a wider range of capacitance. As the capacitance changes from $0.4 \mathrm{pF}$ to $1 \mathrm{pF}$ (a $250 \%$ variation), $R_{f} C_{f}$ only changes from $31.4 \mathrm{~ns}$ to $29.6 \mathrm{~ns}$ (a change of $6 \%$ ). As the capacitance changes from $0.5 \mathrm{pF}$ to $0.7 \mathrm{pF}$ (a $40 \%$ change), the simulated variation in the $R_{f} C_{f}$ product is less than $1 \%$.

\begin{tabular}{|c|c|c|}
\hline $\begin{array}{r}\text { Tuning } \\
* \text { Loo }\end{array}$ & $\begin{array}{c}\text { Table } 1 \\
\text { Simulation } \\
\text { Does Not Co }\end{array}$ & $\begin{array}{l}\text { Results } \\
\text { nverge }\end{array}$ \\
\hline $\begin{array}{c}\mathrm{C}_{1}=\mathrm{C}_{\mathrm{f}} \\
(\mathrm{pF})\end{array}$ & $\begin{array}{c}\mathrm{R}_{\mathrm{f}} \mathrm{C}_{\mathrm{f}}(\mathrm{ns}) \\
\text { for } \mathrm{I}_{\mathrm{R}}=5 \mu \mathrm{A}\end{array}$ & $\begin{array}{l}\mathrm{R}_{\mathrm{f}} \mathrm{C}_{\mathrm{f}}(\mathrm{ns}) \\
\mathrm{I}_{\mathrm{R}}=1 \mu \mathrm{A}\end{array}$ \\
\hline 1.10 & $*$ & $*$ \\
\hline 1.00 & 29.60 & 29.60 \\
\hline 0.90 & 29.88 & 29.79 \\
\hline 0.80 & 30.08 & 29.92 \\
\hline 0.70 & 30.24 & 30.10 \\
\hline 0.60 & 31.62 & 30.24 \\
\hline 0.50 & 34.90 & 30.40 \\
\hline 0.45 & 54.45 & 30.56 \\
\hline 0.40 & $*$ & 31.40 \\
\hline 0.30 & $*$ & 33.00 \\
\hline 0.25 & $*$ & 42.50 \\
\hline
\end{tabular}

\section{CONCLUSION}

A TA detection and recovery scheme for a read channel has been presented. The AC coupler and its tuning loop were described in detail. A Gm cell in the tuning loop is used here to control the on-resistance of a triode MOS transistor in the AC coupler. This approach differs from [4], where a Gm cell in the tuning loop was used to control an identical $\mathrm{Gm}$ cell in a filter. A key advantage of the proposed tuning circuit is that it saves IC area and power. Had a replica of the circuit in Fig. 3 been used for tuning, more than twice the power would have been dissipated as the high-bandwidth opamp in Fig. 3 consumes much more power than all the circuits in Fig. 5. With $I_{R}=1 \mu \mathrm{A}$, the simulated variation in the filter time constant is less than $6 \%$ when there is a $250 \%$ variation in the capacitance, and the time-constant variation is $1 \%$ for a $40 \%$ capacitance variation. While the tuned time constant is slightly less than the design target, the time-constant accuracy is acceptable in a disk drive.

\section{REFERENCES}

[1] S.E. Stupp, M.A. Baldwinson, P. McEwen, T.M. Crawford, and C.T. Rogers, "Thermal Asperity Trends," IEEE Tran. on Magnetics, pp. 752-757, March 1999.
[2] K.G. Ashar, Magnetic Disk Drive Technology, Heads, Media, Channel, Interface, and Integration, IEEE Press, 1997.

[3] K.R. Laker and W.M.C. Sansen, Design of Analog Integrated Circuits and Systems, McGraw-Hill, 1994.

[4] J. Silva-Martinez, M.S.J. Steyaert, and W. Sansen, "A 10.7-MHz 68-dB SNR CMOS Continuous-Time Filter with On-Chip Automatic Tuning", IEEE J. Solid-State Circuits, pp. 1843-1853, Dec. 1992.

\section{FIGURES}

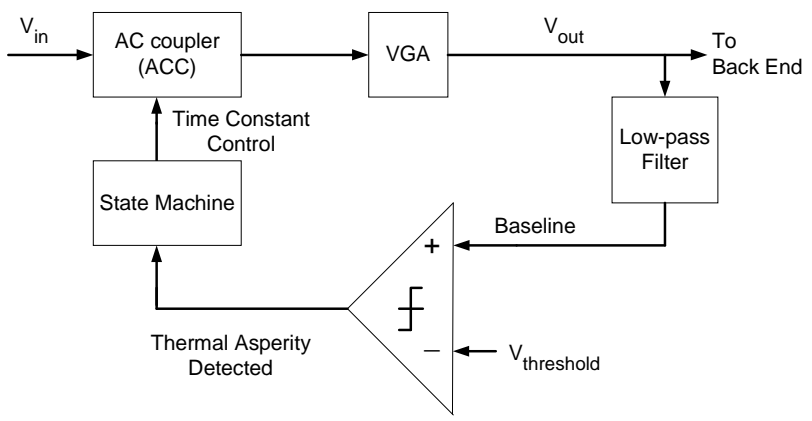

Fig. 1. Block Diagram of a Read Channel Front End.

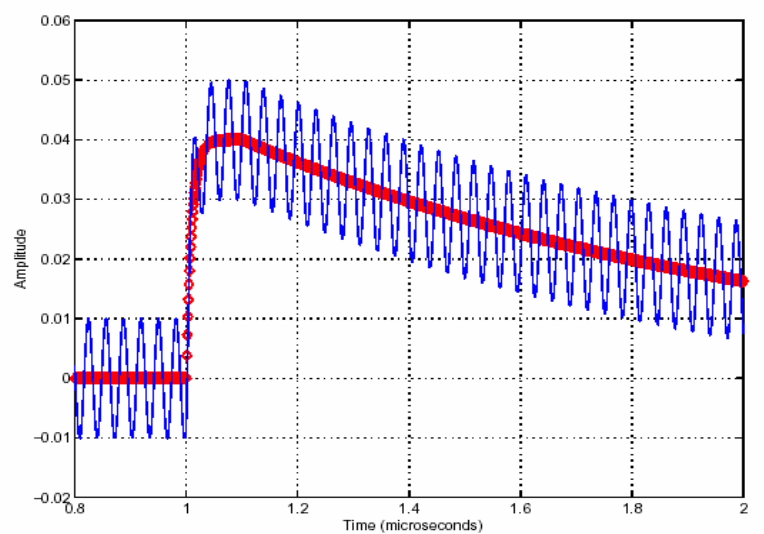

Fig. 2. A TA event: $V_{\text {in }}$ (thin line) and its baseline (thick line) are plotted.

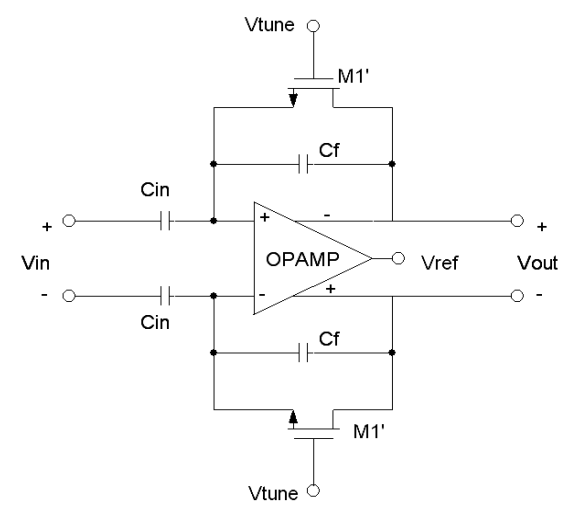

Fig. 3. AC coupler. 


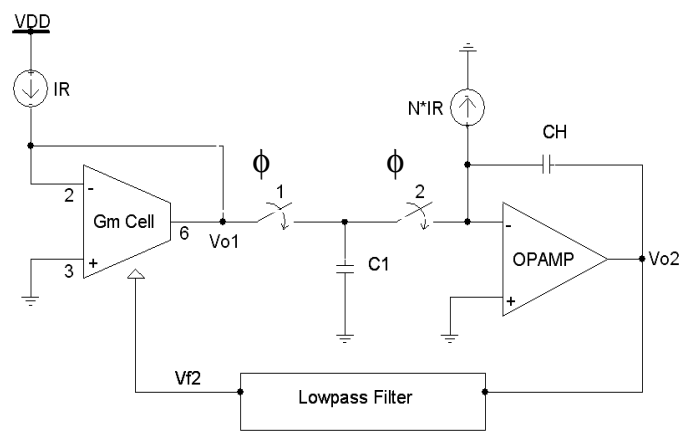

Fig. 4. Simplified Tuning Loop.

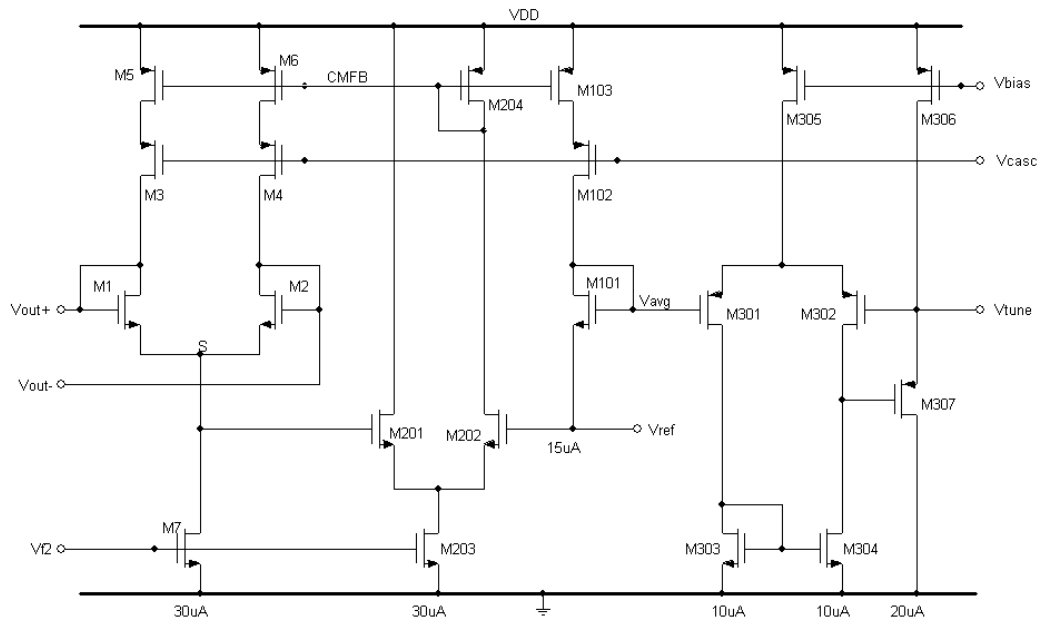

Fig. 6. Gm Cell. Bias currents are along the bottom.

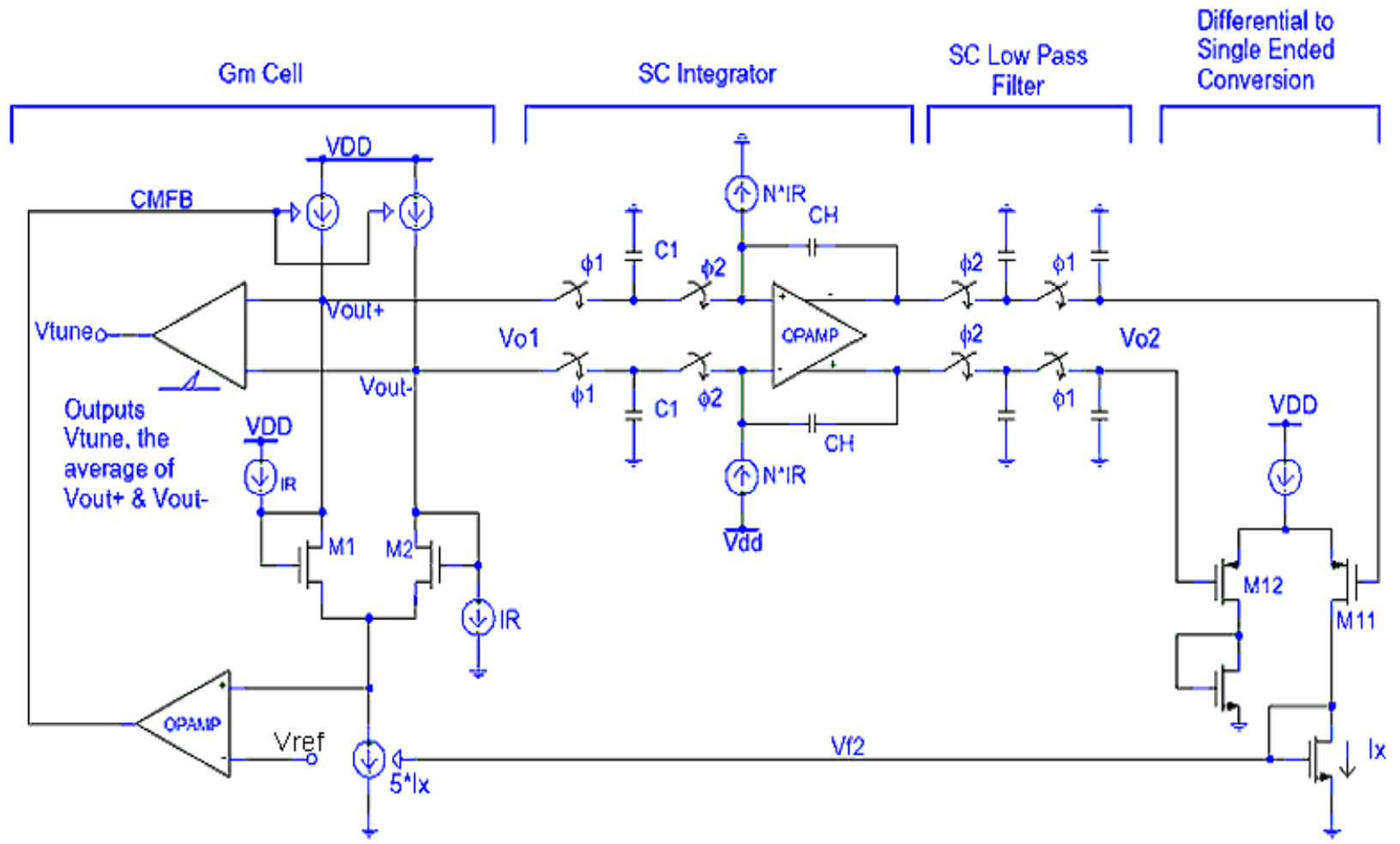

Fig. 5. Detailed Block Diagram of the Tuning Loop. $V_{D D}=3.3 \mathrm{~V}$. 